\title{
Significance of Parafibromin Expression in Laryngeal Squamous Cell Carcinomas
}

Inju Cho $\cdot$ Mija Lee $\cdot$ Sharon Lim Ran Hong

Department of Pathology, Chosun University School of Medicine, Gwangju, Korea

Received: December 30, 2015

Revised: April 15, 2016

Accepted: April 24, 2016

\section{Corresponding Author}

Ran Hong, MD, PhD

Department of Pathology,

Chosun University School of Medicine,

309 Pilmun-daero, Dong-gu,

Gwangju 61452, Korea

Tel: +82-62-230-6356

Fax: +82-62-234-4584

E-mail: nanih@chosun.ac.kr

\begin{abstract}
Background: Parafibromin is a product of the tumor suppressor gene that has been studied as a potential indicator of tumor aggressiveness in the parathyroid, breast, colorectum, and stomach. However, the clinical significance and potential function of parafibromin expression in head and neck squamous cell carcinomas remain largely unknown. The aim of this study was to evaluate the expression of parafibromin in laryngeal squamous cell carcinoma (LSCC) and to verify its potential as a biomarker of tumor behavior. Methods: Parafibromin expression was evaluated in 30 cases of LSCC using immunohistochemistry. The correlations between parafibromin expression and clinicopathologic parameters were investigated. Results: Parafibromin expression was positive in 15 cases (50\%) and negative in 15 cases (50\%). Tumor size and T stage showed a statistically significant inverse relationship with parafibromin expression $(p=.028$ and $p<.001$, respectively). Parafibromin expression was not associated with age, sex, lymph node metastasis, tumor differentiation, or tumor location. There was no statistically significant relationship between parafibromin expression and progression-free survival in the patients $(p>.05)$. Conclusions: Our results indicate that the downregulation or loss of parafibromin expression can be employed as a novel marker of tumor progression or aggressiveness in LSCC.
\end{abstract}

Key Words: Parafibromin; Carcinoma, squamous cell; Epithelial-mesenchymal transition
Laryngeal cancer is one of the most common malignancies in the head and neck area and the 11th most common cancer worldwide, with a high mortality rate and poor prognosis. About $85 \%-90 \%$ of all malignant tumors in the larynx are squamous cell carcinoma. ${ }^{1-3}$ Primary malignancies of the head and neck region other than squamous cell carcinoma are typically salivary gland tumors and neuroendocrine tumors, with rare cases of cartilaginous and soft tissue sarcomas. ${ }^{1}$ The incidence of laryngeal squamous cell carcinoma (LSCC) has gradually increased in recent years. Invasion and metastasis are the main factors affecting a patient's overall survival and quality of life. ${ }^{2}$ Major progress has been achieved in the treatment of laryngeal cancer. Organ-sparing protocols that combine radiation, chemotherapy, and surgery are the standard therapy for advanced laryngeal cancer. ${ }^{1}$ Although the treatment of laryngeal cancer has been evolving, there are currently no markers for assessing prognosis or tumor behavior in LSCC. Therefore, it is worthwhile to investigate biomarkers for understanding tumor behavior and for guidance to optimal treatment.

Parafibromin is a protein encoded by the tumor suppressor gene hyperthyroidism 2 (HRPT2). Its mutation leads to hyper- parathyroidism-jaw-tumor syndrome, an autosomal dominant disease characterized by parathyroid adenoma or carcinoma, fibro-osseous tumors of the maxilla and mandible, renal anomalies, and uterine tumors. ${ }^{4-6}$ The HRPT2 gene is located on human chromosome 1q31.2, which is composed of 17 exons and spans $18.5 \mathrm{~kb}$ in the genome. The gene encodes a $2.7-\mathrm{kb}$ transcript that is translated into a $60-\mathrm{kD}, 531$-amino acid parafibromin protein. ${ }^{7,8}$ Along with its role as an oncosuppressor, parafibromin binds to RNA polymerase II as part of polymeraseassociated factor 1 (Paf1), causing repression of $\beta$-catenin-mediated transcription and transcriptional repression of c-myc. ${ }^{5,9}$ Zhang et al. ${ }^{10}$ documented that the overexpression of parafibromin results in anti-proliferative properties through the inhibition of colony formation and cellular proliferation and induces cell cycle arrest in the G1 phase.

Various non-morphological techniques including reverse transcriptase polymerase chain reaction and northern and western blotting have suggested that parafibromin is ubiquitously expressed. $^{11}$

Immunohistochemically, a high expression of parafibromin has been found in hepatocytes, renal cortex tubules, cells at the base 
of the gastric glands, and the pars intermedia of the hypophysis. ${ }^{11}$ Hyperparathyroidism-jaw-tumors-syndrome and sporadic parathyroid carcinoma show loss of nuclear immunoreactivity of parafibromin. ${ }^{12}$ Selvarajan $e t$ al. ${ }^{13}$ showed inverse correlations between parafibromin expression and tumor size, pathologic stage, and lymphovascular invasion of breast carcinomas. Subsequent investigations have suggested that down-regulated parafibromin expression is involved in tumor pathogenesis, growth, invasion, and metastasis and might be a novel biomarker indicating a poor prognosis in gastric and colorectal carcinomas. ${ }^{14,15}$ Zhang et al. ${ }^{5}$ demonstrated that the down-regulated expression of parafibromin protein might have a negative impact on apoptosis and suppressing cell cycle progression, proliferation, migration, invasion, and the epithelial mesenchymal transition (EMT), suggesting its important role in the pathogenesis, differentiation, and metastasis of head and neck squamous cell carcinoma (HNSCC). Parafibromin has recently been shown to be expressed in squamous cell carcinoma of the head and neck. ${ }^{5}$ However, research on the relationships between parafibromin and clinicopathologic parameters in LSCC has been limited. The purpose of our study was to investigate the expression of parafibromin in LSCC and to identify its clinicopathologic significance.

\section{MATERIALS AND METHODS}

\section{Patient and tissue samples}

This study was composed of 30 cases of LSCC patients who underwent surgery with/without neck dissection at the Department of Otorhinolaryngology at Chosun University Hospital (Gwangju, South Korea) from January 2003 to December 2008. Twenty cases of total laryngectomy, seven cases of partial laryngectomy, and three cases of cordectomy were included. For comparative analysis, 10 cases with normal mucosal tissue of the larynx were also included.

\section{Histopathological evaluation}

For each case, the clinical records and tissue slides were retrospectively re-evaluated by two pathologists (I.C. and R.H.). The examined tissues were prepared in $10 \%$ neutral formalin fixation, paraffin-embedded, and sectioned in 4-5 $\mu \mathrm{m}$ thickness. Hematoxylin and eosin-stained sections were examined using a light microscope (Olympus BX51, Olympus Corporation, Tokyo, Japan). We re-evaluated the histological diagnosis, tumor size, $\mathrm{T}$ stage, and lymph node metastasis. Tumor staging was evaluated according to the 2005 World Health Organization Classification of Head and Neck Tumors. For immunohisto- chemical (IHC) analysis, a representative area of the tumor was selected, and slides were prepared.

\section{IHC evaluation}

IHC study was performed according to the manufacturer's instruction. For IHC analysis of parafibromin, formalin-fixed and paraffin-embedded blocks were sectioned at $4 \mu \mathrm{m}$ thickness and mounted on precoated glass slides, which were then deparaffinized in xylene. Endogenous peroxidase was blocked with a blocking reagent (sodium chloride-citrate, Ventana Medical Systems, Tucson, AZ, USA). The IHC analysis of parafibromin (1:50, sc-33638, rabbit polyclonal, Santa Cruz Biotechnology Inc., Santa Cruz, CA, USA) was carried out using the NexES autoimmunostainer (Ventana Medical Systems), and immunolocalization was performed using the mouse ImmunoCruz Staining System (sc2050, Santa Cruz Biotechnology Inc.). The slides with the primary antibody were incubated for 32 minutes (1:50, parafibromin). For the secondary detection method, the UltraviewUniversal DAB detection kit (cat. No. 760500, Ventana Medical Systems) was used. Slides were then counterstained with hematoxylin (cat. No. 7602021, Ventana Medical Systems). Scoring was performed by two pathologists, and nuclear stain-

Table 1. Clinical and histopathologic features of the patients

\begin{tabular}{|c|c|}
\hline Clinical and histological feature & No. of patients (\%) \\
\hline Age, mean (yr) & 66.5 \\
\hline \multicolumn{2}{|l|}{ Gender } \\
\hline Male & $28(93.3)$ \\
\hline Female & $2(6.7)$ \\
\hline \multicolumn{2}{|l|}{ Tumor location } \\
\hline Localized $^{a}$ & $14(47)$ \\
\hline Transglottic & $16(53)$ \\
\hline \multicolumn{2}{|l|}{ Tumor differentiation } \\
\hline Well differentiated & $12(40.0)$ \\
\hline Moderately differentiated & $16(53.3)$ \\
\hline Poorly differentiated & $2(6.7)$ \\
\hline \multicolumn{2}{|l|}{ Tumor size (cm) } \\
\hline$<1$ & $5(16.7)$ \\
\hline 1 to $<2$ & $9(30)$ \\
\hline 2 to $<3$ & $9(30)$ \\
\hline 3 to $<4$ & $3(10)$ \\
\hline$\geq 4$ & $4(13.3)$ \\
\hline \multicolumn{2}{|l|}{ T stage } \\
\hline 1 & $9(30)$ \\
\hline 2 & $4(13.3)$ \\
\hline 3 & $9(30)$ \\
\hline 4 & $8(26.7)$ \\
\hline \multicolumn{2}{|l|}{ Lymph node metastasis } \\
\hline Absent & $17(56.7)$ \\
\hline Present & $13(43.3)$ \\
\hline
\end{tabular}

Tumors localized to supraglottis, glottis or subglottis. 
ing was interpreted as positive for parafibromin. The expression positivity was graded and counted. The staining intensity score was graded as 1, weak; 2, intermediate; and 3, strong. The percentage of tumor cells with positive expression and the staining intensity grade were multiplied to obtain a final score. Of 30 cases, 11 were scored as 0,15 were scored as $>100$ (range, 115 to 260), and four were scored between 0 and 100. Statistical analyses were performed based on a two-tier system of the parafibromin-positive group and -negative group (positive, > 100; negative, $\leq 100$ ).

\section{Statistical analysis}

Statistical evaluation was carried out using the SPSS ver. 21 (IBM Co., Armonk, NY, USA). The chi-square test and Fisher exact test were used to demonstrate the correlations between parafibromin expression and clinicopathologic characteristics. Survival analysis was performed using Kaplan-Meier survival graphs and the $\log$-rank test. A p-value $<.05$ was considered to be statistically significant.

\section{RESULTS}

\section{Clinical and pathological parameters}

The clinicopathologic characteristics of the patients are summarized in Table 1. The mean age was 66.5 years (range, 47 to 81 years), and the male to female ratio was 13.2:1, showing a male predominance. Tumors localized in the supraglottic, glottic, or subglottic regions composed $47 \%$ ( $n=14)$ of the group, and transglottic tumors were found in $53 \%$ of patients $(\mathrm{n}=16)$. The tumors had a size $<2 \mathrm{~cm}$ in 14 cases ( $46.7 \%$ ) and a size $\geq 2 \mathrm{~cm}$ in 16 cases $(53.3 \%)$. The tumor stage was $\mathrm{T} 1$ in nine cases $(30.0 \%)$ and $\mathrm{T} 2,3$, or 4 in 21 cases $(70.0 \%)$. Lymph node metastasis was present in $43.3 \%$ of the cases $(n=13)$.

\section{The relationships between parafibromin expression and clinicopathologic parameters}

Among the 30 cases, parafibromin expression was positive in 15 cases (50\%) and negative in 15 cases (50\%) (Fig. 1A-D, Table 2). Among cases with positive parafibromin nuclear staining,

Table 2. The relationships between parafibromin expression and clinicopathologic parameters

\begin{tabular}{|c|c|c|c|}
\hline \multirow{2}{*}{ Clinicopathologic parameter } & \multicolumn{2}{|c|}{ Parafibromin expression } & \multirow{2}{*}{$p$-value } \\
\hline & Negative $(n=15,50 \%)$ & Positive $(n=15,50 \%)$ & \\
\hline \multicolumn{4}{|l|}{ Tumor location } \\
\hline Localizeda & 4 & 10 & .066 \\
\hline Transglottic & 11 & 5 & \\
\hline Tumor differentiation & & & .583 \\
\hline Well differentiated & 7 & 5 & \\
\hline Moderately differentiated & 7 & 9 & \\
\hline Poorly differentiated & 1 & 1 & \\
\hline \multicolumn{4}{|l|}{ Tumor size (cm) } \\
\hline $0-0.9$ & 0 & 5 & $.04^{b}$ \\
\hline $1.0-1.9$ & 4 & 5 & \\
\hline $2.0-2.9$ & 5 & 4 & \\
\hline $3.0-3.9$ & 2 & 1 & \\
\hline$\geq 4.0$ & 4 & 0 & \\
\hline \multicolumn{4}{|l|}{ Tumor size $(\mathrm{cm})^{c}$} \\
\hline$<2$ & 4 & 10 & $.028^{b}$ \\
\hline$\geq 2$ & 11 & 5 & \\
\hline T stage & & & $.015^{b}$ \\
\hline $\mathrm{T} 1$ & 0 & 9 & \\
\hline T2 & 4 & 0 & \\
\hline T3 & 6 & 3 & \\
\hline T4 & 5 & 3 & \\
\hline \multicolumn{4}{|l|}{ T stage ${ }^{d}$} \\
\hline $\mathrm{T} 1$ & 0 & 9 & $<.001^{b}$ \\
\hline $\mathrm{T} 2,3$, and 4 & 15 & 6 & \\
\hline \multicolumn{4}{|l|}{ Lymph node metastasis } \\
\hline Absent & 8 & 9 & .713 \\
\hline Present & 7 & 6 & \\
\hline
\end{tabular}

aTumors localized to supraglottis, glottis, or subglottis; ’ Indicates $p<0.05$; ' Subdivided tumor size; 'S Subdivided T stage. 
a subset of cases showed parafibromin immunoreactivity at the periphery of the tumor cell nest (Fig. 1C). There was a statistically significant inverse relationship between parafibromin expression and $\mathrm{T}$ stage $(\mathrm{p}=.015)$. Interestingly, when we subdivided T stage into a T1 group and a T2, 3, or 4 group, the loss of parafibromin expression was associated with the latter $(\mathrm{p}<$ .001) (Table 2). Tumor size and parafibromin expression were also inversely correlated $(\mathrm{p}=.028)$ (Table 2 ). Parafibromin expression was not associated with other clinicopathologic factors such as age, sex, lymph node metastasis, tumor differentiation, or tumor location (Table 2). The follow-up periods of LSCC patients ranged from 2 to 136 months (median, 23 months). During the follow-up period, there was no disease-specific death among the patients. Eight patients experienced distant metastasis or recurrence of the cancer, and progression-free survival (PFS) ranged from 2 to 136 months (median, 21.5 months). The survival curve using the Kaplan-Meier method indicated no statistically significant relationship between parafibromin expression and
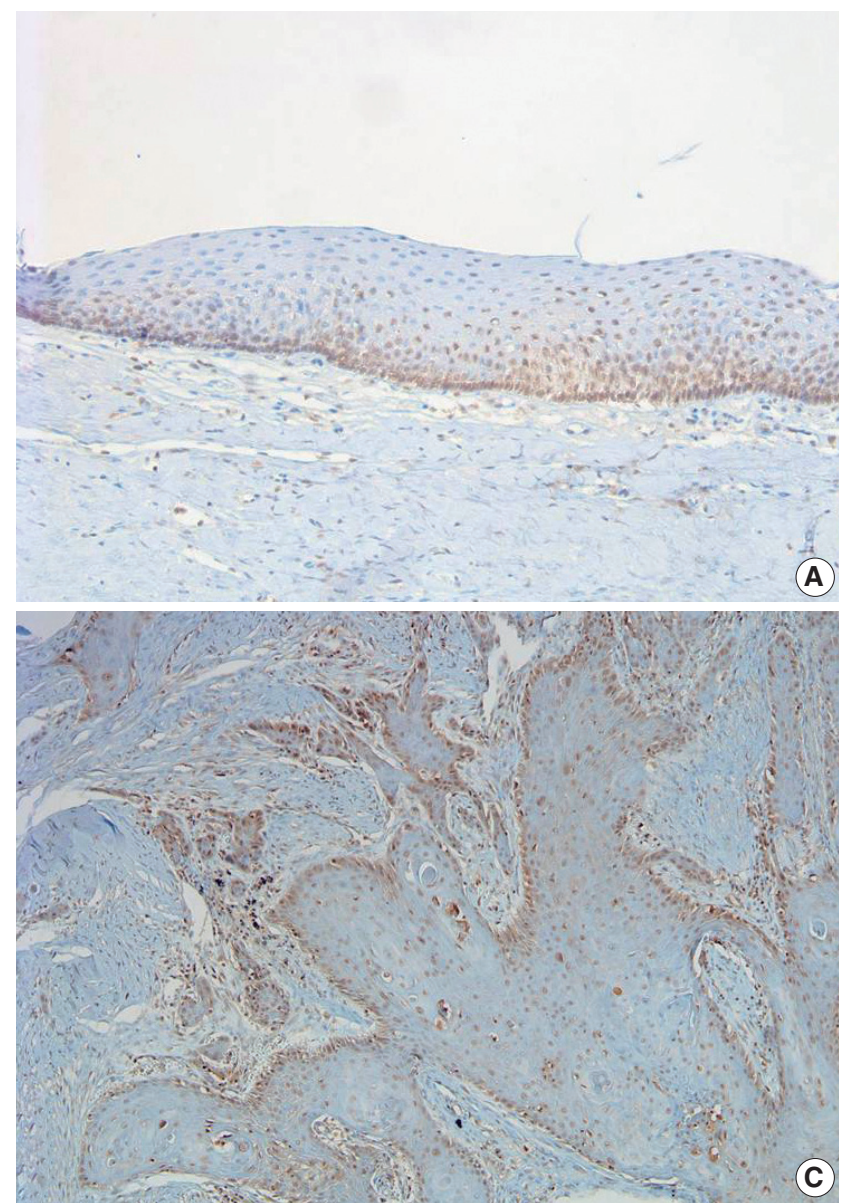

PFS in the patients ( $p>.05)$ (Fig. 2).

\section{DISCUSSION}

Parafibromin is a protein product of the tumor suppressor gene that has been studied as a potential indicator of tumor aggressiveness in many organs, notably the parathyroid, breast, colorectum, and stomach. ${ }^{13-16}$ However, the clinical significance and potential function of parafibromin expression in HNSCC have remained largely unknown. One study by Zhang et al. ${ }^{5}$ investigated the role of parafibromin expression in tumorigenesis and the progression of HNSCC, along with its value as a prognostic marker. The overall 5-year survival rate of HNSCC is about 40\%-50\%, despite advancements in therapy. ${ }^{5}$ The prognosis of laryngeal cancer varies considerably according to the anatomical site of origin. For example, glottic cancer has a better prognosis than supraglottic and subglottic cancer. ${ }^{17}$ Until now, prevention and early diagnosis with function-preserving treatment have been
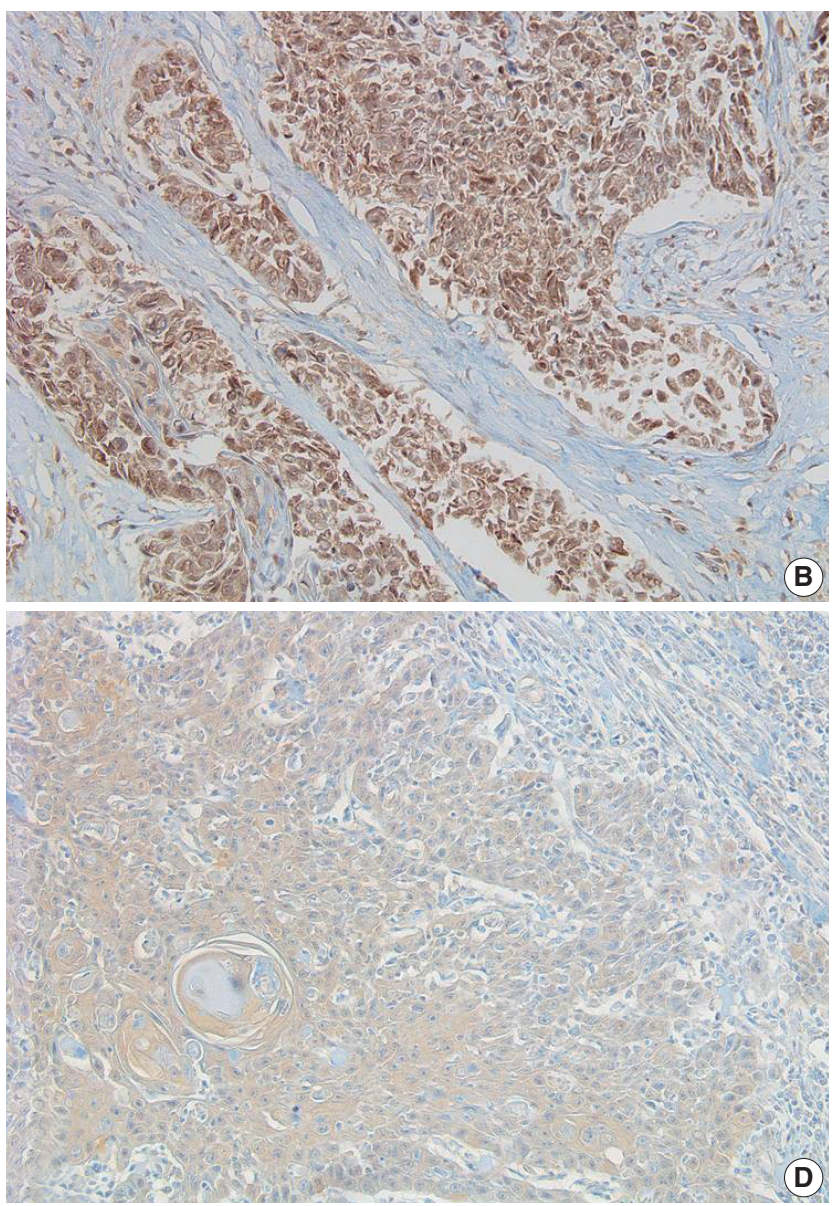

Fig. 1. Parafibromin expression in squamous cell carcinoma of the larynx. (A) Parafibromin expressed in the basal layer of normal laryngeal mucosa. (B) Parafibromin-positive staining is expressed in the nuclei of tumor cells. (C) Occasionally, parafibromin positivity is observed in the periphery of the tumor nest. (D) Representative image of parafibromin-negative staining. 


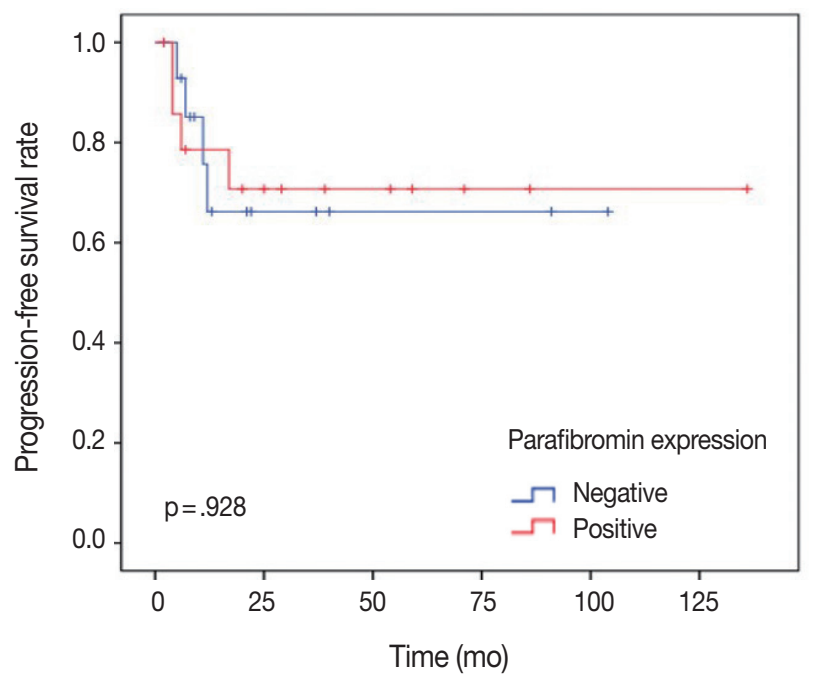

Fig. 2. Kaplan-Meier curves of progression-free survival in laryngeal squamous cell carcinoma patients according to parafibromin expression status. There is no statistically significant relationship between parafibromin expression and the progression-free survival of the patients $(p>.05)$.

considered to be the most effective approach. ${ }^{3}$ The aim of this study was to evaluate the expression of parafibromin in LSCC and verify its potential as a biomarker of tumor behavior. We evaluated the relationships between parafibromin staining and clinicopathologic parameters. Our results demonstrated that parafibromin expression has an inverse correlation with $\mathrm{T}$ stage and tumor size, indicating that downregulation or loss of parafibromin expression is involved in tumor progression or aggressiveness. Our results are in line with other studies on parafibromin expression in different organs. Zheng et al..$^{14}$ observed that tumor size, depth of invasion, lymph node metastasis, clinicopathologic staging, and poor prognosis were inversely correlated with parafibromin in colorectal carcinomas. Zheng et al. ${ }^{15}$ also reported parafibromin expression to be associated with tumor size, depth of invasion, lymph node metastasis, lymphatic invasion, International Union against Cancer staging, and Lauren's classification in gastric carcinomas. Selvarajan et al. ${ }^{13} \mathrm{ob}-$ served that parafibromin expression was inversely correlated with tumor size in 163 cases of breast carcinomas.

Recent research on HNSCC conducted by Zhang et al. showed that parafibromin expression was negatively correlated with lymph node metastasis and TNM staging but positively correlated with human papillomavirus positivity. However, other clinicopathological characteristic such as tumor status were not associated with parafibromin expression. Our results in LSCC revealed that parafibromin expression was inversely correlated with T stage (T1 or T2, 3, and 4) ( $\mathrm{p}<.05)$. Stage $\mathrm{T} 1$ involves tu- mor limited to one subsite of origin with normal vocal cord mobility. A T stage of 2, 3, or 4 involves tumor invading adjacent subsites of origin with impaired vocal cord mobility.

In addition to tumor size and volume, which are encompassed by the $\mathrm{T}$ stage, advanced stage disease with vocal cord fixation might be a significant parameter of tumor aggressiveness. These results also explain the role of parafibromin expression in invasion, migration, and EMT. In vitro experiments have documented that parafibromin overexpression suppressed the migration and invasion of transfected cells by upregulating E-cadherin expression and downregulating the expression of matrix metalloproteinase (MMP)-2, MMP-9, and Slug. ${ }^{5}$ Thus, the loss or downregulation of parafibromin might promote invasion and migration by upregulating Snail, Slug, Zeb1, and Zep2 during EMT. However, unlike in other studies, we did not find significant relationship between parafibromin expression and lymph node metastasis or PFS. Considering our small sample size, this might not represent characteristics of LSCC but rather reflect the limited number of cases. This is a preliminary evaluation that requires support from additional studies based on larger groups with extended follow-up periods and clinicopathologic parameters.

Here, we analyzed parafibromin expression and clinicopathologic features in laryngeal cancer, revealing positive links between loss of parafibromin and tumor size and T stage. Our results suggest that parafibromin expression can be employed as a novel marker of tumor aggressiveness in LSCC. Based on this data, we suggest that parafibromin can be used to guide the individualized treatment of laryngeal cancer.

\section{Conflicts of Interest}

No potential conflict of interest relevant to this article was reported.

\section{Acknowledgments}

This study was supported by research funds from Chosun University, Republic of Korea, 2014.

\section{REFERENCES}

1. Genden EM, Ferlito A, Silver CE, et al. Evolution of the management of laryngeal cancer. Oral Oncol 2007; 43: 431-9.

2. Li L, Wang J, Gao L, Gong L. Expression of paxillin in laryngeal squamous cell carcinoma and its prognostic value. Int J Clin Exp Pathol 2015; 8: 9232-9.

3. Marioni G, Marchese-Ragona R, Cartei G, Marchese F, Staffieri A. 
Current opinion in diagnosis and treatment of laryngeal carcinoma. Cancer Treat Rev 2006; 32: 504-15.

4. Karaarslan S, Yaman B, Ozturk H, Kumbaraci BS. Parafibromin staining characteristics in urothelial carcinomas and relationship with prognostic parameters. J Pathol Transl Med 2015; 49: 389-95.

5. Zhang Z, Yang XF, Huang KQ, et al. The clinicopathological significances and biological functions of parafibromin expression in head and neck squamous cell carcinomas. Tumour Biol 2015; 36: 9487-97.

6. Newey PJ, Bowl MR, Thakker RV. Parafibromin: functional insights. J Intern Med 2009; 266: 84-98.

7. Carpten JD, Robbins CM, Villablanca A, et al. HRPT2, encoding parafibromin, is mutated in hyperparathyroidism-jaw tumor syndrome. Nat Genet 2002; 32: 676-80.

8. Wang PF, Tan MH, Zhang C, Morreau H, Teh BT. HRPT2, a tumor suppressor gene for hyperparathyroidism-jaw tumor syndrome. Horm Metab Res 2005; 37: 380-3.

9. Yart A, Gstaiger M, Wirbelauer C, et al. The HRPT2 tumor suppressor gene product parafibromin associates with human PAF1 and RNA polymerase II. Mol Cell Biol 2005; 25: 5052-60.

10. Zhang C, Kong D, Tan MH, et al. Parafibromin inhibits cancer cell growth and causes G1 phase arrest. Biochem Biophys Res Commun 2006; 350: 17-24.

11. Porzionato A, Macchi V, Barzon L, et al. Immunohistochemical as- sessment of parafibromin in mouse and human tissues. J Anat 2006; 209: 817-27.

12. Gill AJ, Clarkson A, Gimm O, et al. Loss of nuclear expression of parafibromin distinguishes parathyroid carcinomas and hyperparathyroidism-jaw tumor (HPT-JT) syndrome-related adenomas from sporadic parathyroid adenomas and hyperplasias. Am J Surg Pathol 2006; 30: 1140-9.

13. Selvarajan S, Sii LH, Lee A, et al. Parafibromin expression in breast cancer: a novel marker for prognostication? J Clin Pathol 2008; 61: 64-7.

14. Zheng HC, Wei ZL, Xu XY, et al. Parafibromin expression is an independent prognostic factor for colorectal carcinomas. Hum Pathol 2011; 42: 1089-102.

15. Zheng HC, Takahashi H, Li XH, et al. Downregulated parafibromin expression is a promising marker for pathogenesis, invasion, metastasis and prognosis of gastric carcinomas. Virchows Arch 2008; 452: $147-55$.

16. Howell VM, Haven CJ, Kahnoski K, et al. HRPT2 mutations are associated with malignancy in sporadic parathyroid tumours. J Med Genet 2003; 40: 657-63.

17. Licitra L, Bernier J, Grandi C, et al. Cancer of the larynx. Crit Rev Oncol Hematol 2003; 47: 65-80. 